\title{
TMS over the superior temporal sulcus affects expressivity evaluation of portraits
}

\author{
Chiara Ferrari $^{1} \cdot$ Susanna Schiavi ${ }^{1} \cdot$ Zaira Cattaneo $^{1,2}$ \\ Published online: 8 August 2018 \\ (C) Psychonomic Society, Inc. 2018
}

\begin{abstract}
When viewing a portrait, we are often captured by its expressivity, even if the emotion depicted is not immediately identifiable. If the neural mechanisms underlying emotion processing of real faces have been largely clarified, we still know little about the neural basis of evaluation of (emotional) expressivity in portraits. In this study, we aimed at assessing - by means of transcranial magnetic stimulation (TMS) - whether the right superior temporal sulcus (STS) and the right somatosensory cortex (SC), that are important in discriminating facial emotion expressions, are also causally involved in the evaluation of expressivity of portraits. We found that interfering via TMS with activity in (the face region of) right STS significantly reduced the extent to which portraits (but not other paintings depicting human figures with faces only in the background) were perceived as expressive, without, though, affecting their liking. In turn, interfering with activity of the right SC had no impact on evaluating either expressivity or liking of either paintings' category. Our findings suggest that evaluation of emotional cues in artworks recruit (at least partially) the same neural mechanisms involved in processing genuine biological others. Moreover, they shed light on the neural basis of liking decisions in art by art-naïve people, supporting the view that aesthetic appreciation relies on a multitude of factors beyond emotional evaluation.
\end{abstract}

Keywords STS $\cdot$ TMS $\cdot$ Artworks $\cdot$ Emotion

In recent years there has been an increasing interest in the neural mechanisms mediating visual art appreciation (for reviews, see Boccia et al., 2016; Chatterjee \& Vartanian, 2016; Pearce et al., 2016; Pelowski, Markey, Forster, Gerger, $\&$ Leder, 2017). Within this field of investigation, a few studies have directly compared neural responses with observation or evaluation of artworks (e.g., a painting, a sculpture) versus photographs depicting a similar content (e.g., an everyday object, a face, a human body), finding, indeed, some relevant differences between artistic and nonartistic stimuli. For instance, prosocial/altruistic behavior elicited by viewing

Electronic supplementary material The online version of this article (https://doi.org/10.3758/s13415-018-0630-4) contains supplementary material, which is available to authorized users.

Chiara Ferrari

chiara.ferrari@unimib.it

1 Department of Psychology, University of Milano-Bicocca, Piazza dell' Ateneo Nuovo,1, 20126 Milano, Italy

2 IRCCS Mondino Foundation, Pavia, Italy photographs of real people suffering is attenuated when a similar scene is depicted in a painting (Kesner \& Horáček, 2017). Furthermore, core regions of the social brain (such as the ventromedial prefrontal cortex) respond to photographed bodies but not to bodies represented in artworks, the latter, in turn, triggering more activity in the parietal cortex, possibly reflecting increased general visuospatial coding related to the artistic status of the image (Lutz et al., 2013). Moreover, viewing images of sculptures selected from masterpieces of Classical and Renaissance art compared with corresponding photos of young athletes induced stronger activation of the right dorsal anterior insula - this activation being interpreted by the authors as the hedonic signature of aesthetic experience when viewing artworks (Di Dio, Canessa, Cappa, \& Rizzolatti, 2011). Overall, these findings fit well with the hypothesis that the artistic status of a painting (or of a sculpture) may somehow bias its processing, inducing in the observer an "aesthetic" orientation rather than a "pragmatic" one, with different brain circuits recruited (Cupchik, Vartanian, Crawley, \& Mikulis, 2009; see also Lacey et al., 2011).

In this framework, we were interested in investigating whether focusing on the expression of a portrayed face would 
trigger activation in the same brain regions that are known to be important when dealing with real faces. Indeed, we are often captured by the expressivity of a portray, even if the emotion depicted is not immediately identifiable (think, just to refer to famous examples, of the Girl With a Pearl Earring or the Mona Lisa). The view of (biological) faces showing an emotional expression activates a complex network of subcortical structures (such as the amygdala and the insula; see Atkinson \& Adolphs, 2011) and cortical regions, including the superior temporal sulcus (STS), motor and premotor areas, and the somatosensory cortex (SC; Cohen Kadosh, Henson, Kadosh, Johnson, \& Dick, 2010; Engell \& Haxby, 2007; Fox, Moon, Iaria, \& Barton, 2009; Narumoto, Okada, Sadato, Fukui, \& Yonekura, 2001; Wang et al., 2017; Winston, O’Doherty, \& Dolan, 2003). Accordingly, transcranial magnetic stimulation (TMS) delivered over the (right) STS impairs participants' ability to discriminate (Pitcher, 2014) and evaluate the intensity of facial emotional expressions (Paracampo, Pirruccio, Costa, Borgomaneri, \& Avenanti, 2018) and may indirectly modulate - when applied to posterior sectors - the amygdala's activation (Pitcher, Japee, Rauth, \& Ungerleider, 2017). Indeed, STS (specifically, its posterior sector) seems to be deputed to code facial muscles configurations (i.e., action units) essential for the interpretation and categorization of emotions (Srinivasan, Golomb, \& Martinez, 2016). Moreover, STS is connected with the frontoparietal mirror system (Casile, 2013; Iacoboni et al., 2001; Molenberghs, Brander, Mattingley, \& Cunnington, 2010), critical for embodied mechanisms of emotion recognition (e.g., Perry et al., 2017; Wood, Rychlowska, Korb, \& Niedenthal, 2016). The SC would be particularly important for embodied mechanisms of emotion recognition, as suggested by the reduced intensity of facial mimicry responses (measured through electromyography) to videos of people smiling following TMS over this region (Korb et al., 2015). In line with this, both real lesions in patients and "virtual" lesions of right SC induced by TMS in healthy individuals have been found to interfere with processing of facial emotional expressions (Adolphs, Damasio, Tranel, Cooper, \& Damasio, 2000; Pitcher, Garrido, Walsh, \& Duchaine, 2008; Pourtois et al., 2004). Interestingly, SC may also play a role in more "sophisticated" social inferences, as suggested by the finding that TMS over (right) SC reduces participants' ability to decide whether a smiling individual is feeling authentic amusement or not (Paracampo, Tidoni, Borgomaneri, di Pellegrino, \& Avenanti, 2017).

In this study, we directly investigated whether interfering with SC and STS activity via TMS affects the perceived expressivity of a portrait, and whether this affects the liking of that particular artwork. Our hypothesis is that deciding about the expressivity of a portrait should recruit the same neural circuits that are involved in processing facial expressions in real individuals (but see Di Dio et al., 2011; Kesner \&
Horáček, 2017; Lutz et al., 2013) and, therefore, that TMS over face-sensitive sectors of the STS and the SC should affect participants' judgments. Moreover, since (decisions about) liking a painting may rely — at least in parton the same brain regions involved in sensory information processing (Cattaneo et al., 2015; Cattaneo, Schiavi, Silvanto, \& Nadal, 2017; Vartanian \& Goel, 2004), interfering with STS and SC activity may affect liking beyond expressivity evaluation.

\section{Method}

\section{Participants}

Thirty-six neurologically healthy right-handed Italian students ( 7 males, $M_{\text {age }}=22.8$ years, $S D=1.7$ ), with no previous training or special interest in art, participated in the TMS study. Prior to the experiment, each participant filled in a questionnaire to evaluate compatibility with TMS (translated from Rossi, Hallett, Rossini, \& Pascual-Leone, 2011). Thirty participants ( 11 males, $M_{\text {age }}=22.17$ years, $S D=1.46$ ), with no previous training or special interest in art and not taking part in the TMS experiment, participated in a pilot study aimed to select the stimuli to be used in the main TMS experiment (see below). Participants were treated in accordance with the Declaration of Helsinki.

\section{Material}

Stimuli consisted of 80 reproductions of paintings from the 15 th to the 20th century: half were portraits (in which a single face clearly occupied the foreground of the frame) and half (control stimuli) were paintings depicting human figures whose faces were not in the foreground and occupied only a little portion of the frame. In the portraits we used, faces were portrayed in a frontal pose or slightly turned to one side, with both eyes visible and gaze oriented as the face. Paintings depicting human figures (with faces visible only in the background) were chosen as control stimuli rather than landscapes or still-life compositions because evaluation of artworks with biological and nonbiological content might rely on different criteria (e.g., Massaro et al., 2012), and we wanted to maintain the task as close as possible for the two sets of paintings. Figure 1 shows examples of the two categories of paintings. Although paintings were the work of renowned artists, they were relatively unknown pieces, in line with previous research (e.g., Cela-Conde et al., 2009; Cela-Conde et al., 2004).

The 80 paintings used in the TMS experiments were selected from a larger database of 220 paintings presented in a pilot study. Participants of the pilot study were required to rate the expressivity (or emotional intensity) and liking of this larger set on a 1 to 9 Likert scale ( $1=$ I find it not expressive at all/I 

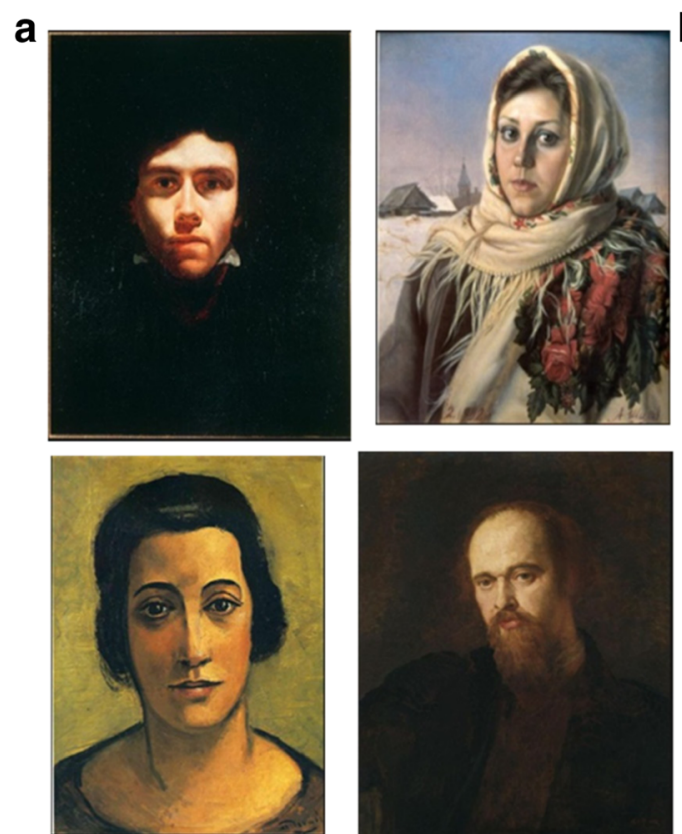

Fig. 1. Examples of portraits (a) and nonportraits (b) used in the TMS experiment. Portraits (from upper left in clockwise order): Portrait of Eugene Delacroix, by Théodore Géricault, 1818-1819; Russian Beauty, by Alexander Shilov, 1992; Dante Gabriel Rossetti, by George Frederic Watts, 1871; Portrait of Madame Carco, by André Derain, 20th century. Nonportraits (from upper left in clockwise order): The Wheat
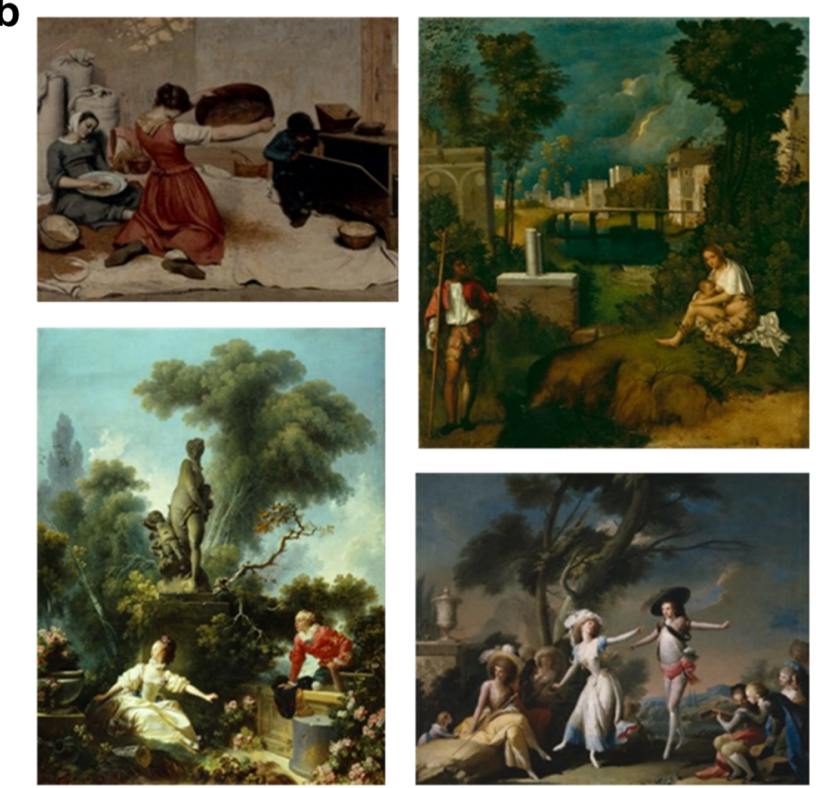

Sifters, by Gustave Courbet, 1854-1855; The Tempest, by Giorgione, 1505; El bolero, by José Camarón Boronat, 1785; The Progress of Love, by Jean-Honore Fragonard, 1771. (All paintings shown in this figure are free from copyright at http://commons.wikimedia.org/ and/or http://www.wikiart.org)

trial started with a fixation cross presented for $2,500 \mathrm{~ms}$ on a white background. This was followed by a $250 \mathrm{~ms}$ white screen after which a painting (subtending approximately 10 $\times 10$ degrees of visual angle) was presented in the central field of view. The painting remained visible until participants' response (see below). Participants were randomly assigned to two different task conditions. In the expressivity task, participants were instructed to classify the painting as "expressive" or "not expressive." Participants were told that although all paintings may convey an emotion, they had to indicate whether they found that specific painting as overall expressive or not. In the liking task, participants indicated whether they liked or did not like the painting. For both task conditions, the instructions referred to evaluation of the painting as a whole, and not to evaluation of the face or of the scene depicted. For both tasks, it was stressed that there was no correct or wrong answer, since the judgment was entirely subjective. Participants responded by left/right key pressing with their right index and middle finger, and they were encouraged to respond as fast as possible. Response-key assignment was counterbalanced across participants. Note that although previous studies using body shape stimuli presented for less than $200 \mathrm{~ms}$ have found reliable liking judgments (e.g., Cazzato, Mele, \& Urgesi, 2014, 2016), we preferred to maintain paintings visible until response to allow more proper processing of the paintings' content (see also Cattaneo et al., 2015; Cattaneo et al., 2017; Graham, Pallett, Meng, \& Leder, 2014; Leder, 


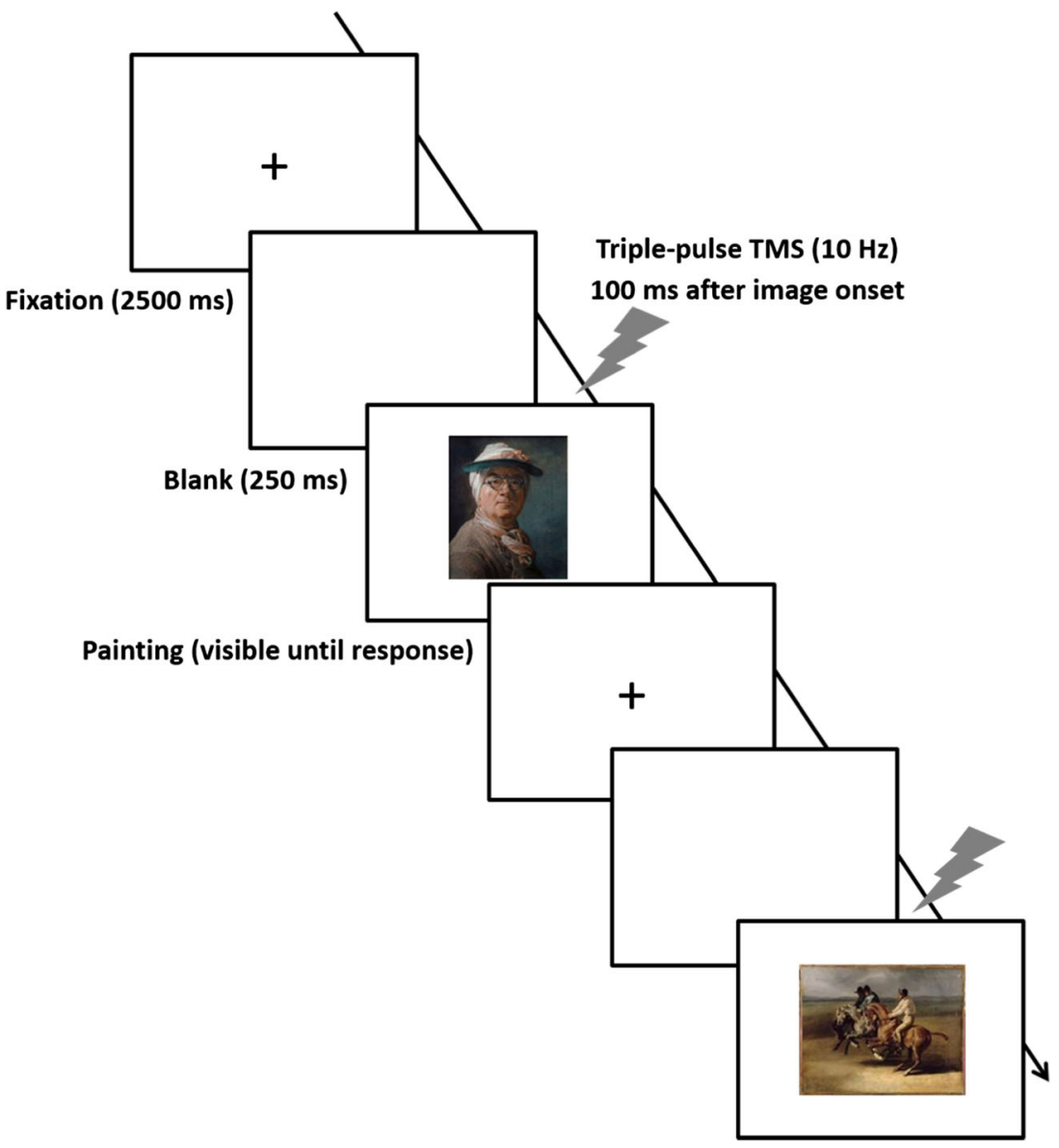

Fig. 2. Schematic overview of an experimental trial showing a portrait painting (Self-Portrait Wearing Glasses, by Jean-Baptiste-Siméon Chardin, 1775) and a nonportrait painting (The Horse Race, by Théodore Géricault, 1820-1824). Each trial started with a fixation cross on a white background, after which a blank screen $(250 \mathrm{~ms})$ appeared, followed by the painting (centrally presented). Participants had to indicate

Gerger, Dressler, \& Schabmann, 2012). In both tasks, the same 80 artworks (40 portraits and 40 nonportraits) were presented in random order. Participants performed each block three times, once for each TMS site (see below), for a total of 240 trials. A short training session of eight trials (with paintings not used in the experimental trials) was performed before the experiment to familiarize with the task. The order of cortical sites stimulation was counterbalanced across participants. The software EPrime 2.0 (Psychology Software Tools, Pittsburgh, PA) was used for stimulus presentation, data collection, and TMS triggering.

\section{Transcranial magnetic stimulation}

TMS was delivered using a Magstim Rapid ${ }^{2}$ stimulator (Magstim Co Ltd., Whitland, UK) connected to a 70-mm butterfly coil at a fixed intensity of $60 \%$ of the maximum stimulator output. A fixed intensity of stimulation was used whether they found the painting overall expressive or not or whether they liked it or not, depending on the task condition they were assigned to. 10 $\mathrm{Hz}$ triple-pulse TMS was applied over the SC, STS, or over the vertex (control site) $100 \mathrm{~ms}$ after the image onset. (All paintings shown in this figure are free from copyright at http://commons.wikimedia.org/ and/or http://www.wikiart.org/)

in many prior studies targeting nonmotor regions (e.g., Bona, Cattaneo, \& Silvanto, 2015; Cattaneo et al., 2014; Ferrari, Lega, et al., 2016; Ferrari, Vecchi, Todorov, \& Cattaneo, 2016; Prime, Vesia, \& Crawford, 2009), including STS and SC (Pitcher, 2014; Pitcher et al., 2008). Targeted sites were the right STS, right SC, and the vertex (control site). We targeted the STS and SC in the right hemisphere in light of consistent evidence suggesting a right lateralization in the response of these regions to emotional faces (e.g., Adolphs et al., 2000; Pourtois et al., 2004). The vertex was localized as the point falling half the distance between the nasion and the inion on the same midline. The STS and the SC were localized by means of stereotaxic navigation on individual estimated magnetic resonance images (MRI) obtained through a 3-D warping procedure fitting a high-resolution MRI template with the participant's scalp model and craniometric points (Softaxic, EMS, Bologna, Italy). This procedure has been proven to ensure a global localization accuracy of roughly 5 $\mathrm{mm}$, a level of precision closer to that obtained using 
individual MRIs than to what can be achieved using other localization methods (Carducci \& Brusco, 2012), and it has been successfully used in many prior studies (e.g., Balconi \& Ferrari, 2013a, 2013b). Anatomical Talairach coordinates of the right posterior STS were $x=52, y=-48, z=8$, corresponding to the sector of STS that showed the strongest response to faces displaying an emotional expression compared to those displaying a neutral expression in a previous fMRI study (Engell \& Haxby, 2007). Talairach coordinates of right SC were $x=44, y=-12, z=48$, and were taken from a previous fMRI study by Winston et al. (2003) in which this region was significantly more active during discrimination of emotion versus gender of faces. These exact coordinates for right SC have also been used in a following TMS study by Pitcher et al. (2008), finding that that this face-sensitive sector (but not a finger-sensitive sector) of right SC plays a causal role in facial emotion discrimination (Pitcher et al., 2008). Triple-pulse TMS $(10 \mathrm{~Hz})$ was delivered $100 \mathrm{~ms}$ after the onset of the painting, given prior TMS evidence showing that contribution of both SC and STS in perceiving facial expressions takes place in a time window ranging from $100 \mathrm{~ms}$ to $200 \mathrm{~ms}$ from the stimulus onset (Pitcher et al., 2008; Pourtois et al., 2004).

\section{Results}

The number of positive (i.e., "I find this painting expressive" or "I like this painting") responses was calculated for each participant in each block and converted into a percentage score (see Fig. 3). Similarly, mean response latencies (RT) were calculated for each participant in each TMS condition (see Fig. 4). Trials in which participants' RT were \pm 3 standard deviations above or below their own average response time were excluded from the analyses ( $1.0 \%$ of trials were excluded following this criterion). The dependent variables were analyzed via repeated-measures ANOVAs with task (expressivity vs. liking) as between-subjects factor and painting type (portrait vs. nonportrait) and TMS site (SC, STS, and vertex) as within-subjects factors.

The analysis on yes/no responses revealed no significant main effect of painting type, $F(1,34)<1, p=.41$; TMS site, $F(2,68)<1, p=.68$; or task, $F(1,34)<1, p=.83$. The interaction of Painting Type $\times$ TMS Site $\times$ Task reached significance, $F(2,68)=5.14, p=.008, \eta_{\mathrm{p}}^{2}=.13$. No other interactions reached significance (all $p \mathrm{~s}>.09$ ). The threeway interaction of Painting Type $\times$ TMS Site $\times$ Task was further investigated via a repeated-measures ANOVA with TMS site and painting type as within-subjects variables for the expressivity task and the liking task, separately. For the liking task, neither painting type, $F(1,17)<1, p=.35$, nor TMS site, $F(2,34)<1, p=.80$, nor the interaction of Painting Type $\times$ TMS, $F(2,34)=1.36, p=.27$, reached significance. For the expressivity task, the main effect of painting type, $F(1,17)<1, p=.66$, and the main effect of TMS site, $F(2,34)=1.05, p=.36$, were not significant. In turn, the interaction of Painting Type $\times$ TMS Site reached significance, $F(2,34)=5.68, p=.007, \eta_{\mathrm{p}}^{2}=.25$. Post hoc comparisons (Bonferroni-Holm correction applied) showed that TMS over STS significantly reduced the number of portraits perceived as expressive compared with TMS over the vertex, $t(17)=2.79, p$ $=.039, d=.66$, and compared with TMS over the SC, $t(17)=$ $2.61, p=.036, d=.62$ (see also Fig. S1 in the Supplementary Materials). No differences were found between TMS over the vertex and TMS over SC, $t(17)<1, p=.99$. In turn, TMS did not affect perceived expressivity of nonportrait paintings (all post hoc comparisons, $p \mathrm{~s}>.24$ ).

The analysis on mean response latencies for both positive and negative responses showed a significant main effect of painting type, $F(1,34)=27.34, p<.001, \eta_{\mathrm{p}}^{2}=.45$, with slightly faster responses to portraits $(M=756 \mathrm{~ms}, S D=199)$ than to nonportrait paintings $(M=800 \mathrm{~ms}, S D=222)$. No

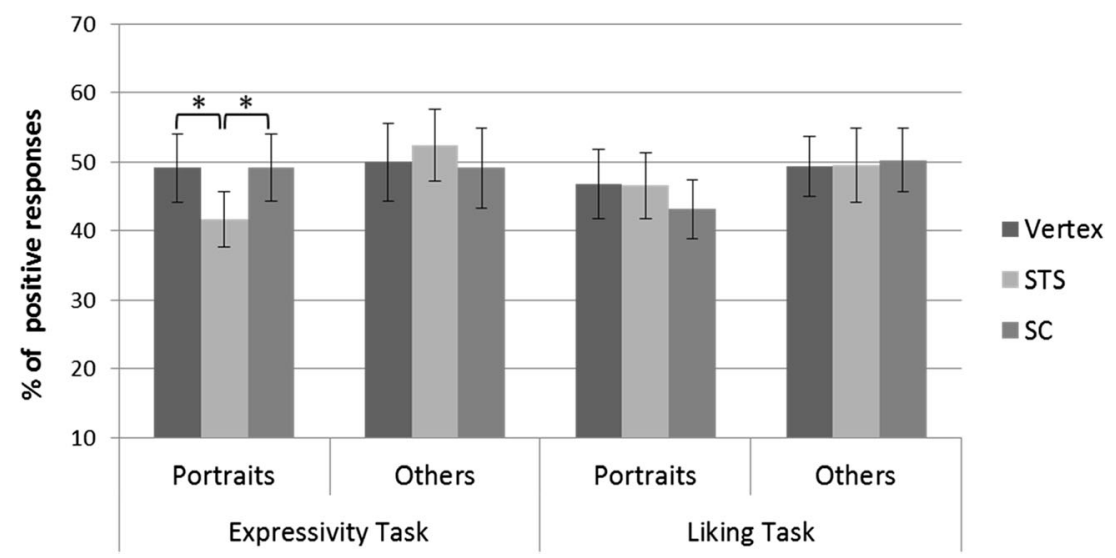

Fig. 3. Percentage of positive responses as a function of TMS condition (vertex, SC, STS), painting type (portrait vs. nonportrait) and task (expressivity task vs. liking task). TMS over STS significantly decreased the number of paintings perceived as expressive compared

with both TMS over the vertex and TMS over the SC. In turn, TMS did not affect liking decisions. Error bars indicate $\pm 1 S E M$. Asterisks indicate a significant difference ( $p<.05$, Bonferroni-Holm corrected) across TMS conditions 


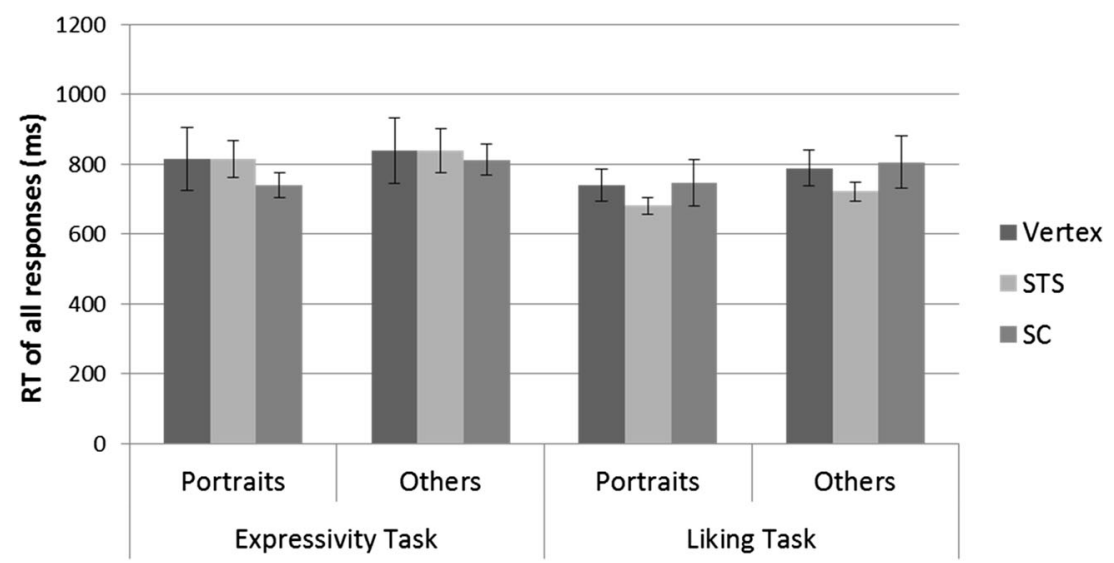

Fig. 4. Response latencies as a function of TMS condition (vertex, SC, STS), painting type (portrait vs. non-portrait) and task (expressivity vs. liking decision). TMS did not affect RT. Error bars indicate \pm 1 SEM

other main effects or interactions reached significance $(p s>$ .08 ; see Fig. 4). Note that a similar analysis selectively performed on positive responses yielded the same pattern of results - that is, a significant main effect of painting type, $F(1$, 34) $=9.00, p=.005, \eta_{\mathrm{p}}^{2}=.21$, and no other significant main effects ( $p=.60$ for task; $p=.68$ for TMS site) or interactions (all $p \mathrm{~s}>.13$ ).

\section{Discussion}

Our results show that interfering via TMS with activity in the face region of the right superior temporal sulcus (STS) significantly reduced the extent to which portraits (but not other paintings depicting human figures with faces only in the background) were perceived as expressive, without, though, affecting liking decisions. In turn, TMS over the face region of the right somatosensory cortex (SC) had no impact on evaluating either expressivity or liking of either paintings' category.

There is evidence that processing of artworks recruit neural circuits that only partially overlap with those involved in processing nonartistic images with similar content (de Gelder et al., 2017; Di Dio et al., 2011; Kesner \& Horáček, 2017; Lacey et al., 2011; Lutz et al., 2013). Focusing on portraits, a study carried out on a set of portraits painted by famous Western artists found that the latter tended to draw human faces with statistical properties that deviate from the face photographs and approximate the scale invariant, fractal-like properties of complex natural scenes (Redies, Hänisch, Blickhan, \& Denzler, 2007). Moreover, artists would tend to overregularize the structure of portraits compared with the structure of real faces (Schweinhart \& Essock, 2013) and to manipulate lighting conditions (e.g., low vs. high contrast) of portraits to obtain more extreme emotional expressions (see Sakuta, Kanazawa, \& Yamaguchi, 2014). If on the one hand these artistic techniques would make the faces aesthetically more pleasing, or somehow closer to the expected canonical ideal
(Redies et al., 2007; Schweinhart \& Essock, 2013), on the other hand they may render the portrayed faces more "artificial" and hence less prone - at the neurophysiological level - to be processed as real genuine others (e.g., Lutz et al., 2013; see also Graham, Stockinger, \& Leder, 2013). Our data shed light on this issue by showing that STS, a key brain region in facial emotion recognition (e.g., Cohen Kadosh et al., 2010; Engell \& Haxby, 2007; for a review see Atkinson \& Adolphs, 2011), is causally involved in processing expressivity of portrayed faces.

The lack of TMS effects over expressivity evaluation of nonportrait paintings may appear at odds with prior evidence showing that STS is also involved in processing biologically salient stimuli other than faces, such as human bodies moving or in postures implying motion, or in (bodily) action prediction (e.g., Avenanti, Candidi, \& Urgesi, 2013; Barraclough, Xiao, Oram, \& Perrett, 2006; Dasgupta, Tyler, Wicks, Srinivasan, \& Grossman, 2017; Grossman, Battelli, \& Pascual-Leone, 2005; Jokisch, Daum, Suchan, \& Troje, 2005; Makris \& Urgesi, 2014). However, in our experiment, we specifically targeted a sector of STS that strongly responded to faces in a prior neuroimaging study (Engell \& Haxby, 2007). A combined TMS/fMRI study has recently shown that the transient disruption of the face-selective region of the right posterior STS reduced neural responses to faces but not to bodies or objects in the anterior STS (and amygdala), suggesting that face and body's related information is processed via different functionally pathways within STS (Pitcher et al., 2017). Moreover, it is possible that the STSTMS effect on expressivity evaluation of portraits partially depended on stimulation also affecting processing of gaze direction, to which STS is quite sensitive (Carlin \& Calder, 2013; see also Boyarskaya, Sebastian, Bauermann, Hecht, \& Tüscher, 2015), and that is known to affect perceived intensity of emotional faces (e.g., Adams \& Kleck, 2005; Milders, Hietanen, Leppänen, \& Braun, 2011). Although this may be possible, it is worth noting that neuronal clusters responding 
to gaze and emotional expressions in STS only partially overlap (Engell \& Haxby, 2007; Pourtois et al., 2004). In our nonportrait set, faces were also present, but they occupied a relatively small portion of the background of the frame, likely being of insufficient salience to trigger a significant response in the face-sensitive sector of STS. Also, in considering the lack of STS stimulation on evaluation of paintings depicting human figures (with no faces in the foreground) it is worth noting that in a prior neuroimaging study comparing brain activation for sculptures versus photos of real bodies, STS was found to activate in both conditions, but significantly more so in the case of real bodies (Di Dio et al., 2011).

Contrary to our hypothesis, interfering with activity in the face region of the somatosensory cortex (SC) did not affect evaluation of portraits' expressivity. This finding seems inconsistent with prior evidence showing that embodied mechanisms relying on the activity of SC are relevant for processing of emotional expressions in real faces (Adolphs et al., 2000; Pitcher et al., 2008; Rychlowska et al., 2014; Winston et al., 2003). In interpreting this null finding, though, it should be considered that other prior TMS studies also failed to find an effect of right SC stimulation on face emotion discrimination (Mattavelli, Cattaneo, \& Papagno, 2011) or found effects only for specific emotions (e.g., fear but not happiness; see Pourtois et al., 2004). These effects have been interpreted as suggesting that the excitability threshold of somatosensory regions to show a TMS-related interference may vary depending on the emotion category, being higher for emotions whose recognition requires strong activation of internal somatic representations (Pourtois et al., 2004). In our task, we selected paintings of moderate expressivity (see Material section) to allow TMS to affect participants' responses: indeed, dichotomic classification of stimuli too polarized in terms of the feature to be evaluated would be little vulnerable to TMS modulation (e.g., Devlin \& Watkins, 2007; Robertson et al., 2003). It is hence possible that the portrayed faces we used were not sufficiently expressive to elicit activation in SC. Moreover, the continuous shift of attention between the analysis of the content and the aesthetic features (e.g., style) occurring during the perception of an artwork (Kesner \& Horáček, 2017; see also Cupchik et al., 2009; Marković, 2010, 2012), may reduce the strength of the emotional and empathic responses toward the depicted objects, further weakening (in the case of our task) the involvement of SC. Accordingly, although null findings always need to be interpreted with caution, our results may also suggest that, contrary to real faces, portraits do not elicit embodiment in sensory cortices.

Consistent evidence suggests that category-specific areas of the visual system, such as extrastriate body area, the motion-sensitive area V5, the lateral occipital cortex (LO), and the fusiform gyrus, are not only engaged in the visual processing of artworks but also contribute to their aesthetic evaluation (e.g., Calvo-Merino, Urgesi, Orgs, Aglioti, \&
Haggard, 2010; Cattaneo et al., 2015; Cattaneo et al., 2017; Chatterjee, Thomas, Smith, \& Aguirre, 2009; Chatterjee \& Vartanian, 2014; for reviews, Boccia et al., 2016; Kirsch, Urgesi, \& Cross, 2016; Vartanian, \& Skov, 2014). In particular, prior brain stimulation studies have found that interfering with LO - a key region for object and shape processing (GrillSpector, 2003) - during aesthetic appreciation selectively reduced the evaluation of representational paintings, leaving the appreciation of abstract paintings unaffected (Cattaneo et al., 2015). In turn, TMS over V5 significantly reduced the extent of motion perceived in both figurative and abstract paintings, but selectively diminished liking for the latter category (Cattaneo et al., 2017). These results were interpreted as suggesting that because there are multiple factors feeding into aesthetic appreciation - liking of an artwork depending on a complex interplay of sensation, emotion, and cognition (Chatterjee \& Vartanian, 2014; Pearce et al., 2016; Pelowski et al., 2017) - selectively interfering with processing of one factor (such as implied motion or content-related features) may not significantly affect the level of appreciation. Our findings here seem to support this interpretation. Emotional expressivity, or the extent to which an artwork is moving, is certainly an important factor in individuals' liking of paintings (Gernot, Pelowski, \& Leder, 2018; Leder et al., 2012), as it is also shown by our pilot study in which emotional and aesthetic evaluations of the same set of paintings positively correlated. Still, the reduction of perceived expressivity through STS stimulation failed to affect liking of portraits. A key point here may also be the question we posed to participants. In our task, we did not ask for liking of the portrayed face (or scene) but rather for liking of the artwork per se, liking decisions about content and artwork per se relying on different mechanisms (e.g., Hayn-Leichsenring, Kloth, Schweinberger, \& Redies, 2013; Redies, 2015; see also Gernot et al., 2018). We may speculate that in judging expressivity participants focused specifically on the face or the content depicted (as the most intuitive strategy to guess expressivity by art-naïve observers), whereas in deciding about liking other factors beyond the depicted content (such as style, colors) may have weighted more. The weight in driving liking decisions of factors such as stylistic and content-related features, the empathetic engagement with the depicted content and/or with the intention/performance of the artist (see Umiltà, Berchio, Sestito, Freedberg, \& Gallese, 2012), but also the perceived "artistry" of a painting per se (Gerger, Leder, \& Kremer, 2014) may vary significantly depending on the task requirement and the type of stimuli used. Further investigation is needed to disentangle the contribution of these factors and their neural substrates in aesthetic evaluation.

TMS did not affect response latencies in our experiment. Selective effects of TMS on the decision output and not on RTs are not uncommon in TMS literature and have been reported in several prior studies (e.g., Cattaneo et al., 2017; 
Pitcher, Charles, Devlin, Walsh, \& Duchaine, 2009; Pitcher et al., 2008). Differential effects of TMS on different dependent variables such as accuracy, sensitivity measures, or reaction times may depend on the type of stimuli used, on the task demand and difficulty (Devlin \& Watkins, 2007). The only effect we observed on reaction times in our study was related to the painting category, with participants taking longer to evaluate nonportraits than portraits in both the expressivity and liking conditions. This may have depended on participants finding overall easier to judge a single face in the foreground (with no other critical elements present) compared with a more complex scene involving more characters and possibly requiring further cognitive elaboration to understand the depicted situation. In this regard, one may wonder whether the different response latencies for the two painting categories contributed to the selective TMS effect observed only for the type of paintings eliciting faster decisions (i.e., portraits). However, this is unlikely, since face expressions are encoded in STS already within the first $200 \mathrm{~ms}$ from stimulus presentation (Pitcher, 2014), with our stimulation entirely covering that relevant time window. Moreover, prior evidence has shown that similar stimulation parameters (i.e., triple-pulse $10 \mathrm{~Hz}$ TMS delivered $100 \mathrm{~ms}$ from stimulus onset over prefrontal and parietal sites) affected the evaluation of stimuli taking similar response times than non-portrait paintings in this study (Cattaneo, Lega, Gardelli, et al., 2014).

To conclude, our study demonstrates that the role of STS in processing facial expressions extends to the evaluation of expressivity of portraits. In turn, the somatosensory cortex seems to be less relevant in deciding about emotional intensity of portrayed faces. Our results represent an important step forward in understanding how the brain processes different aspects of visual artworks and suggest that noninvasive brain stimulation is indeed a quite promising technique to shed light on the causal role of different brains regions in artworks evaluation (Cattaneo et al., 2015; Cattaneo, Lega, Flexas, et al., 2014; Cattaneo, Lega, Gardelli, et al., 2014; Cattaneo et al., 2017).

Acknowledgements This work was supported by a PRIN grant (2015WXAXJF) by Italian Ministry of Education, University and Research to Z.C.

\section{References}

Adams, R. B., Jr., \& Kleck, R. E. (2005). Effects of direct and averted gaze on the perception of facially communicated emotion. Emotion, $5(1), 3-11$.

Adolphs, R., Damasio, H., Tranel, D., Cooper, G., \& Damasio, A. R. (2000). A role for somatosensory cortices in the visual recognition of emotion as revealed by three-dimensional lesion mapping. Journal of Neuroscience, 20(7), 2683-2690.

Atkinson, A. P., \& Adolphs, R. (2011). The neuropsychology of face perception: Beyond simple dissociations and functional selectivity.
Philosophical Transactions of the Royal Society of London B: Biological Sciences, 366(1571), 1726-1738.

Avenanti, A., Candidi, M., \& Urgesi, C. (2013). Vicarious motor activation during action perception: Beyond correlational evidence. Frontiers in Human Neuroscience, 7, 185.

Balconi, M., \& Ferrari, C. (2013a). Left DLPFC rTMS stimulation reduced the anxiety bias effect or how to restore the positive memory processing in high-anxiety subjects. Psychiatry Research, 209(3), 554-559.

Balconi, M., \& Ferrari, C. (2013b). Repeated transcranial magnetic stimulation on dorsolateral prefrontal cortex improves performance in emotional memory retrieval as a function of level of anxiety and stimulus valence. Psychiatry and Clinical Neurosciences, 67(4), 210-218.

Barraclough, N. E., Xiao, D., Oram, M. W., \& Perrett, D. I. (2006). The sensitivity of primate STS neurons to walking sequences and to the degree of articulation in static images. Progress in Brain Research, 154, 135-148.

Boccia, M., Barbetti, S., Piccardi, L., Guariglia, C., Ferlazzo, F., Giannini, A. M., \& Zaidel, D. W. (2016). Where does brain neural activation in aesthetic responses to visual art occur? Meta-analytic evidence from neuroimaging studies. Neuroscience \& Biobehavioral Reviews, 60, 65-71.

Bona, S., Cattaneo, Z., \& Silvanto, J. (2015). The causal role of the occipital face area (OFA) and lateral occipital (LO) cortex in symmetry perception. Journal of Neuroscience, 35(2), 731-738.

Boyarskaya, E., Sebastian, A., Bauermann, T., Hecht, H., \& Tüscher, O. (2015). The Mona Lisa effect: Neural correlates of centered and offcentered gaze. Human Brain Mapping, 36(2), 619-632.

Calvo-Merino, B., Urgesi, C., Orgs, G., Aglioti, S. M., \& Haggard, P. (2010). Extrastriate body area underlies aesthetic evaluation of body stimuli. Experimental Brain Research, 204(3), 447-456.

Carducci, F., \& Brusco, R. (2012). Accuracy of an individualized MRbased head model for navigated brain stimulation. Psychiatry Research: Neuroimaging, 203(1), 105-108.

Carlin, J. D., \& Calder, A. J. (2013). The neural basis of eye gaze processing. Current Opinion in Neurobiology, 23(3), 450-455.

Casile, A. (2013). Mirror neurons (and beyond) in the macaque brain: An overview of 20 years of research. Neuroscience Letters, 540, 3-14.

Cattaneo, Z., Lega, C., Ferrari, C., Vecchi, T., Cela-Conde, C. J., Silvanto, J., \& Nadal, M. (2015). The role of the lateral occipital cortex in aesthetic appreciation of representational and abstract paintings: A TMS study. Brain and Cognition, 95, 44-53.

Cattaneo, Z., Lega, C., Flexas, A., Nadal, M., Munar, E., \& Cela-Conde, C. J. (2014a). The world can look better: Enhancing beauty experience with brain stimulation. Social Cognitive and Affective Neuroscience. 9(11), 1713-1721.

Cattaneo, Z., Lega, C., Gardelli, C., Merabet, L. B., Cela-Conde, C. J., \& Nadal, M. (2014b). The role of prefrontal and parietal cortices in aesthetic appreciation of representational and abstract art: A TMS study. NeuroImage, 99, 443-450.

Cattaneo, Z., Schiavi, S., Silvanto, J., \& Nadal, M. (2017). A TMS study on the contribution of visual area V5 to the perception of implied motion in art and its appreciation. Cognitive Neuroscience, 8(1), 5968.

Cazzato, V., Mele, S., \& Urgesi, C. (2014). Gender differences in the neural underpinning of perceiving and appreciating the beauty of the body. Behavioural Brain Research, 264, 188-196.

Cazzato, V., Mele, S., \& Urgesi, C. (2016). Different contributions of visual and motor brain areas during liking judgments of same-and different-gender bodies. Brain Research, 1646, 98-108.

Cela-Conde, C. J., Ayala, F. J., Munar, E., Maestú, F., Nadal, M., Capó, M. A., ... Marty, G. (2009). Sex-related similarities and differences in the neural correlates of beauty. Proceedings of the National Academy of Sciences, 106(10), 3847-3852. 
Cela-Conde, C. J., Marty, G., Maestú, F., Ortiz, T., Munar, E., Fernández, A., ... Quesney, F. (2004). Activation of the prefrontal cortex in the human visual aesthetic perception. Proceedings of the National Academy of Sciences of the United States of America, 101(16), 6321-6325.

Chatterjee, A., Thomas, A., Smith, S. E., \& Aguirre, G. K. (2009). The neural response to facial attractiveness. Neuropsychology, 23(2), $135-143$.

Chatterjee, A., \& Vartanian, O. (2014). Neuroaesthetics. Trends in Cognitive Sciences, 18(7), 370-375.

Chatterjee, A., \& Vartanian, O. (2016). Neuroscience of aesthetics. Annals of the New York Academy of Sciences, 1369(1), 172-194.

Cohen Kadosh, K. C., Henson, R. N., Kadosh, R. C., Johnson, M. H., \& Dick, F. (2010). Task-dependent activation of face-sensitive cortex: An fMRI adaptation study. Journal of Cognitive Neuroscience, 22(5), 903-917.

Cupchik, G. C., Vartanian, O., Crawley, A., \& Mikulis, D. J. (2009). Viewing artworks: Contributions of cognitive control and perceptual facilitation to aesthetic experience. Brain and Cognition, 70(1), 84 91.

Dasgupta, S., Tyler, S. C., Wicks, J., Srinivasan, R., \& Grossman, E. D. (2017). Network connectivity of the right STS in three social perception localizers. Journal of Cognitive Neuroscience, 29(2), 221234.

de Gelder, B., Watson, R., Zhan, M., Diano, M., Tamietto, M., \& Vaessen, M. (2017). Gender-specific brain activation during visual art perception. BioRxiv, 104166. doi:https://doi.org/10.1101/104166

Devlin, J. T., \& Watkins, K. E. (2007). Stimulating language: insights from TMS. Brain, 130, 610-622.

Di Dio, C., Canessa, N., Cappa, S. F., \& Rizzolatti, G. (2011). Specificity of aesthetic experience for artworks: An fMRI study. Frontiers in Human Neuroscience, 5, 139.

Engell, A. D., \& Haxby, J. V. (2007). Facial expression and gaze-direction in human superior temporal sulcus. Neuropsychologia, 45(14), 3234-3241.

Ferrari, C., Lega, C., Vernice, M., Tamietto, M., Mende-Siedlecki, P., Vecchi, T., ... Cattaneo, Z. (2016a). The dorsomedial prefrontal cortex plays a causal role in integrating social impressions from faces and verbal descriptions. Cerebral Cortex, 26, 156-165.

Ferrari, C., Vecchi, T., Todorov, A., \& Cattaneo, Z. (2016b). Interfering with activity in the dorsomedial prefrontal cortex via TMS affects social impressions updating. Cognitive, Affective, \& Behavioral Neuroscience, 16(4), 626-634.

Fox, C. J., Moon, S. Y., Iaria, G., \& Barton, J. J. (2009). The correlates of subjective perception of identity and expression in the face network: An fMRI adaptation study. NeuroImage, 44(2), 569-580.

Gerger, G., Leder, H., \& Kremer, A. (2014). Context effects on emotional and aesthetic evaluations of artworks and IAPS pictures. Acta Psychologica, 151, 174-183.

Gernot, G., Pelowski, M., \& Leder, H. (2018). Empathy, Einfühlung, and aesthetic experience: The effect of emotion contagion on appreciation of representational and abstract art using fEMG and SCR. Cognitive Processing, 19(2), 147-165.

Graham, D., Pallett, P. M., Meng, M., \& Leder, H. (2014). Representation and aesthetics of the human face in portraiture. Art \& Perception, 2(1/2), 75-98.

Graham, D., Stockinger, S., \& Leder, H. (2013). An island of stability: art images and natural scenes - but not natural faces - show consistent aesthetic response in Alzheimer's-related dementia. Frontiers in Psychology, 4, 107

Grill-Spector, K. (2003). The neural basis of object perception. Current Opinion in Neurobiology, 13(2), 159-166.

Grossman, E. D., Battelli, L., \& Pascual-Leone, A. (2005). Repetitive TMS over posterior STS disrupts perception of biological motion. Vision Research, 45(22), 2847-2853.
Hayn-Leichsenring, G. U., Kloth, N., Schweinberger, S. R., \& Redies, C. (2013). Adaptation effects to attractiveness of face photographs and art portraits are domain-specific. I Perception, 4(5), 303-316.

Iacoboni, M., Koski, L. M., Brass, M., Bekkering, H., Woods, R. P., Dubeau, M. C., ... Rizzolatti, G. (2001). Reafferent copies of imitated actions in the right superior temporal cortex. Proceedings of the National Academy of Sciences, 98(24), 13995-13999.

Jokisch, D., Daum, I., Suchan, B., \& Troje, N. F. (2005). Structural encoding and recognition of biological motion: Evidence from event-related potentials and source analysis. Behavioural Brain Research, 157(2), 195-204.

Kesner, L., \& Horáček, J. (2017). Empathy-related responses to depicted people in art works. Frontiers in Psychology, 8.

Kirsch, L. P., Urgesi, C., \& Cross, E. S. (2016). Shaping and reshaping the aesthetic brain: Emerging perspectives on the neurobiology of embodied aesthetics. Neuroscience \& Biobehavioral Reviews, 62, 5668.

Korb, S., Malsert, J., Rochas, V., Rihs, T. A., Rieger, S. W., Schwab, S., ... Grandjean, D. (2015). Gender differences in the neural network of facial mimicry of smiles-An rTMS study. Cortex, 70, 101-114.

Lacey, S., Hagtvedt, H., Patrick, V. M., Anderson, A., Stilla, R., Deshpande, G., ... Sathian, K. (2011). Art for reward's sake: Visual art recruits the ventral striatum. NeuroImage, 55(1), 420-433.

Leder, H., Gerger, G., Dressler, S. G., \& Schabmann, A. (2012). How art is appreciated. Psychology of Aesthetics, Creativity, and the Arts, 6(1), 2.

Lutz, A., Nassehi, A., Bao, Y., Pöppel, E., Sztrókay, A., Reiser, M., ... Gutyrchik, E. (2013). Neurocognitive processing of body representations in artistic and photographic images. NeuroImage, 66, 288 292.

Makris, S., \& Urgesi, C. (2014). Neural underpinnings of superior action prediction abilities in soccer players. Social Cognitive and Affective Neuroscience, 10(3), 342-351.

Marković, S. (2010). Aesthetic experience and the emotional content of paintings. Psihologija, 43(1), 47-64.

Marković, S. (2012). Components of aesthetic experience: aesthetic fascination, aesthetic appraisal, and aesthetic emotion. i-Perception, $3(1), 1-17$.

Massaro, D., Savazzi, F., Di Dio, C., Freedberg, D., Gallese, V., Gilli, G., \& Marchetti, A. (2012). When art moves the eyes: A behavioral and eye-tracking study. PLOS ONE, 7(5), e37285.

Mattavelli, G., Cattaneo, Z., \& Papagno, C. (2011). Transcranial magnetic stimulation of medial prefrontal cortex modulates face expressions processing in a priming task. Neuropsychologia, 49(5), 992-998.

Milders, M., Hietanen, J. K., Leppänen, J. M., \& Braun, M. (2011). Detection of emotional faces is modulated by the direction of eye gaze. Emotion, 11(6), 1456.

Molenberghs, P., Brander, C., Mattingley, J. B., \& Cunnington, R. (2010). The role of the superior temporal sulcus and the mirror neuron system in imitation. Human Brain Mapping, 31(9), 1316-1326.

Narumoto, J., Okada, T., Sadato, N., Fukui, K., \& Yonekura, Y. (2001). Attention to emotion modulates fMRI activity in human right superior temporal sulcus. Cognitive Brain Research, 12(2), 225-231.

Paracampo, R., Pirruccio, M., Costa, M., Borgomaneri, S., \& Avenanti, A. (2018). Visual, sensorimotor and cognitive routes to understanding others' enjoyment: An individual differences rTMS approach to empathic accuracy. Neuropsychologia, 116(Pt. A), 86-98. doi: https://doi.org/10.1016/j.neuropsychologia.2018.01.043

Paracampo, R., Tidoni, E., Borgomaneri, S., di Pellegrino, G., \& Avenanti, A. (2017). Sensorimotor network crucial for inferring amusement from smiles. Cerebral Cortex, 27(11), 5116-5129.

Pearce, M. T., Zaidel, D. W., Vartanian, O., Skov, M., Leder, H., Chatterjee, A., \& Nadal, M. (2016). Neuroaesthetics: The cognitive neuroscience of aesthetic experience. Perspectives on Psychological Science, 11(2), 265-279. 
Pelowski, M., Markey, P. S., Forster, M., Gerger, G., \& Leder, H. (2017). Move me, astonish me... delight my eyes and brain: The Vienna integrated model of top-down and bottom-up processes in art perception (VIMAP) and corresponding affective, evaluative, and neurophysiological correlates. Physics of Life Reviews, 21, 80-125.

Perry, A., Saunders, S. N., Stiso, J., Dewar, C., Lubell, J., Meling, T. R., ... Knight, R. T. (2017). Effects of prefrontal cortex damage on emotion understanding: EEG and behavioural evidence. Brain, 140(4), 1086-1099.

Pitcher, D. (2014). Facial expression recognition takes longer in the posterior superior temporal sulcus than in the occipital face area. The Journal of Neuroscience: The Official Journal of the Society for Neuroscience, 34, 9173-9177.

Pitcher, D., Charles, L., Devlin, J. T., Walsh, V., \& Duchaine, B. (2009). Triple dissociation of faces, bodies, and objects in extrastriate cortex. Current Biology, 19(4), 319-324.

Pitcher, D., Garrido, L., Walsh, V., \& Duchaine, B. C. (2008). Transcranial magnetic stimulation disrupts the perception and embodiment of facial expressions. Journal of Neuroscience, 28(36), 8929-8933.

Pitcher, D., Japee, S., Rauth, L., \& Ungerleider, L. G. (2017). The superior temporal sulcus is causally connected to the amygdala: A combined TBS-fMRI study. Journal of Neuroscience, 37(5), 11561161.

Pourtois, G., Sander, D., Andres, M., Grandjean, D., Reveret, L., Olivier, E., \& Vuilleumier, P. (2004). Dissociable roles of the human somatosensory and superior temporal cortices for processing social face signals. European Journal of Neuroscience, 20(12), 3507-3515.

Prime, S. L., Vesia, M., \& Crawford, J. D. (2009). TMS over human frontal eye fields disrupts trans-saccadic memory of multiple objects. Cerebral Cortex, 20(4), 759-772.

Redies, C. (2015). Combining universal beauty and cultural context in a unifying model of visual aesthetic experience. Frontiers in Human Neuroscience, 9, 218.

Redies, C., Hänisch, J., Blickhan, M., \& Denzler, J. (2007). Artists portray human faces with the Fourier statistics of complex natural scenes. Network, 18, 235-248.
Robertson, E. M., Theoret, H., \& Pascual-Leone, A. (2003). Studies in cognition: The problems solved and created by transcranial magnetic stimulation. Journal of Cognitive Neuroscience, 15(7), 948-960.

Rossi, S., Hallett, M., Rossini, P. M., \& Pascual-Leone, A. (2011). Screening questionnaire before TMS: An update. Clinical Neurophysiology, 122(8), 1686.

Rychlowska, M., Cañadas, E., Wood, A., Krumhuber, E. G., Fischer, A., $\&$ Niedenthal, P. M. (2014). Blocking mimicry makes true and false smiles look the same. PLOS ONE, 9(3), e90876.

Sakuta, Y., Kanazawa, S., \& Yamaguchi, M. K. (2014). Shedding light on painters' implicit knowledge: The effect of lighting on recognizing expression and facial impressions of a depicted person in portraits. Japanese Psychological Research, 56(3), 288-295.

Schweinhart, A. M., \& Essock, E. A. (2013). Structural content in paintings: Artists overregularize oriented content of paintings relative to the typical natural scene bias. Perception, 42(12), 1311-1332.

Srinivasan, R., Golomb, J. D., \& Martinez, A. M. (2016). A neural basis of facial action recognition in humans. Journal of Neuroscience, 36(16), 4434-4442.

Umiltá, M. A., Berchio, C., Sestito, M., Freedberg, D., \& Gallese, V. (2012). Abstract art and cortical motor activation: An EEG study. Frontiers in Human Neuroscience, 6, 311.

Vartanian, O., \& Goel, V. (2004). Neuroanatomical correlates of aesthetic preference for paintings. Neuroreport, 15(5), 893-897.

Vartanian, O., \& Skov, M. (2014). Neural correlates of viewing paintings: Evidence from a quantitative meta-analysis of functional magnetic resonance imaging data. Brain and Cognition, 87, 52-56.

Wang, S., Yu, R., Tyszka, J. M., Zhen, S., Kovach, C., Sun, S., ... Mamelak, A. N. (2017). The human amygdala parametrically encodes the intensity of specific facial emotions and their categorical ambiguity. Nature Communications, 8, 14821.

Winston, J. S., O'Doherty, J., \& Dolan, R. J. (2003). Common and distinct neural responses during direct and incidental processing of multiple facial emotions. NeuroImage, 20(1), 84-97.

Wood, A., Rychlowska, M., Korb, S., \& Niedenthal, P. (2016). Fashioning the face: Sensorimotor simulation contributes to facial expression recognition. Trends in Cognitive Sciences, 20(3), 227240. 